\title{
Nurses' knowledge about and attitudes toward organ donation in state and private hospitals in Johannesburg, South Africa
}

\author{
K Crymble, ${ }^{1}$ Dip Nurs; H R Etheredge, ${ }^{1} \mathrm{PhD} ;$ J Fabian, ${ }^{1,2} \mathrm{MD} ;$ P Gaylard, ${ }^{3} \mathrm{PhD}$ \\ ${ }^{1}$ Wits Donald Gordon Medical Centre, Johannesburg, South Africa \\ ${ }^{2}$ Department of Internal Medicine, School of Clinical Medicine, Faculty of Health Sciences, University of the Witwatersrand, Johannesburg, South Africa \\ ${ }^{3}$ Data Management and Statistical Analysis (DMSA), Johannesburg, South Africa
}

Corresponding author: H R Etheredge (harriet.etheredge@mediclinic.co.za)

\begin{abstract}
Background. Nurses are intricately involved in organ donation; however, the referral of donors appears to be declining in Johannesburg, South Africa (SA). This may be due to barriers in the referral process.

Objectives. The objectives of this study were to explore nurses' knowledge of the organ donation process and to explore personal beliefs and attitudes around organ donation.

Methods. A quantitative, self-administered questionnaire was completed by nurses in Johannesburg, SA.

Results. A total of 273 nurses participated, of whom most were female and $<50$ years old. The majority of participants (64.2\%) reported positive attitudes, and $63.2 \%$ stated that their personal beliefs about organ donation did not influence the advice they gave to patients. However, only $36.8 \%$ felt confident referring potential donors and $35.8 \%$ felt that referral was within their scope of practice. Most participants (84.5\%) felt that it was the doctor's responsibility to refer donors, but $80.3 \%$ noted that they would refer donors themselves if there was a mandatory referral protocol. Only $61 \%$ of nurses were aware that there was access to a transplant procurement coordinator through their hospitals; however, there was uncertainty regarding the role of the coordinator.

Conclusion. There is an urgent need to clarify the role of nurses in the process of organ donor referral in SA. Although nurses felt positive about organ donation, they expressed uncertainties about referring potential donors. However, if a clear protocol for referral was introduced, the majority of nurses noted that they would willingly follow it. We advocate for the development and implementation of a nationally endorsed protocol for donor referral and for the training of nurses in organ donation in SA.
\end{abstract}

S Afr J Crit Care 2017;33(2):52-57. DOI:10.7196/SAJCC.2017.v33i2.322

As is the case in the rest of the world, the supply of donor organs in South Africa (SA) falls far short of the need for organs. The need to increase solid organ donor rates has spurred a number of innovative transplant programmes such as splitting livers from deceased donors, living donor liver transplants from adult donors to paediatric recipients, ${ }^{[1]}$ and HIVpositive donor to HIV-positive recipient kidney transplants. ${ }^{[2]}$ Such initiatives have generally been driven by motivated academic transplant teams at the hospital level, while there has been little corresponding change in transplant legislation or protocol at the national level, even though protocols for organ donor referral have been incorporated into best practice guidelines internationally. ${ }^{[3]}$ There have been significant advances in transplantation in SA with regard to surgical technique and immunosuppression therapy that have allowed this specialised field of medicine to evolve with outcomes that are internationally comparable in many centres ${ }^{[4]}$ however, this is not supported by a solid regulatory framework that involves good governance procedures, national guidelines and protocols for clinical practice. Previous research in SA suggests that this results in confusion about professional roles, challenging inter-professional interactions and loss of potential donors owing to ambiguities in the referral process. ${ }^{[5]}$

This environment of regulatory uncertainty means that organ donation is viewed differently by different health professionals. Attitudes and opinions depend on the nature of their work, the sector in which they work and the extent to which it is encouraged in their place of work. Transplant procurement coordinators, who are tasked with ensuring organ procurement for all health sectors, are continually faced with the challenge of working in a cross-sector protocol vacuum where organ donation is often a source of uncertainty and moral distress. ${ }^{[5]}$ As a transplant procurement coordinator who has been in the field for 18 years, the principal investigator (PI) of this study had anecdotally experienced substantial challenges to organ donor referral in the Johannesburg health sector. These challenges were particularly related to facilitating the buy-in of nurses to refer potential organ donors in both sectors. The rationale for this study was to further investigate these factors through a formal empirical research project so that evidencebased recommendations for change can be made.

The main aim of this study was to explore knowledge about and attitudes toward organ donation among nurses in Johannesburg, SA.

The specific objectives were to explore nurses' knowledge of the organ donation process and their attitudes toward organ donation.

\section{Methods Sampling}

Sampling was undertaken across state and private sector intensive care units (ICUs), casualty and high-care departments, as well as two transplant units in Johannesburg (one state and the other private). 
In total, 7 sites were sampled, including 3 state sites (Chris Hani Baragwanath Academic Hospital, Helen Joseph Hospital, Charlotte Maxeke Johannesburg Academic Hospital) and 4 private sites (Wits Donald Gordon Medical Centre, Sandton Mediclinic, Life Wilgeheuwel Hospital and Life Flora Clinic). Participants were considered eligible to participate if they were qualified nurses, $>18$ years of age and felt comfortable answering an English questionnaire.

The sampling strategy was specifically designed to obtain a fully representative sample of the two healthcare sectors in SA; the ratio of state to private sector nurses was 2:1. The purpose of this sampling strategy was two-fold: firstly, to account for the fact that the state sector services the healthcare needs of approximately two-thirds of South Africans, and hence it was important that this demographic was reflected in the sampling; secondly, this sampling ratio allowed for measuring knowledge, attitudes and perceptions of organ donation in the state sector in Johannesburg, where donor referrals are observed to be low in comparison with the SA private sector.

\section{Data collection}

Data were collected between July 2015 and March 2016, using a quantitative, structured, self-administered which was carefully designed, piloted and pre-tested prior to sampling. The questionnaire was anonymous and no identifying information was collected. Participation in the study was voluntary, and the study was fully explained to all potential participants by the PI before questionnaires were handed out. Administration of the questionnaires took place during nurses' working hours to ensure that both day and night shift nurses were included.

\section{Sample size}

Based on worst-case (for sample size) estimates of $50 \%$ (for any given question response), a $6 \%$ precision and the $95 \%$ confidence level, a sample size of 267 was required. ${ }^{[6]}$ In total, 396 questionnaires were handed out.

\section{Data analysis}

Data were collected and managed using REDCap (Vanderbilt University, USA) electronic data capture tools hosted at the Faculty of Health Sciences, University of the Witwatersrand. ${ }^{[6]}$

A knowledge score was derived from the six knowledge questions by calculating the sum of correct responses from these questions, thus creating a score with a possible range of $0-6$. A Contactability Indicator was derived from Q10 - 11. It was assumed that the transplant coordinator was contactable by the respondent if either Q10 ('Please write down the name and the telephone number of the coordinator you refer potential donors to. If you don't know, please leave the space empty.) was answered correctly or Q11 (Do you have access to the telephone number for the on-call transplant coordinator if you need it?) was answered affirmatively.

The $\chi^{2}$ test was used to assess the relationship between pairs of categorical variables. Fisher's exact test was used for $2 \times 2$ tables or where the requirements for the $\chi^{2}$ test could not be met. The relationship between knowledge score and categorical variables was assessed by the $t$-test (or analysis of variance $>2$ categories). Where the data did not meet the assumptions of these tests, a non-parametric alternative, the Wilcoxon rank sum test (or the Kruskal-Wallis test for $>2$ categories) was used. Data analysis was carried out using SAS version 9.4 (SAS, USA). The $5 \%$ significance level was used.

This research was approved by the Human Research Ethics Committee of the University of the Witwatersrand (ref. no. M150334). As this was a self-administered questionnaire, consent was implied by nurses choosing to participate. Permission to undertake the study was obtained from the chief executive officers of all participating sites as well as the nursing unit managers of the various sections.

\section{Results}

The overall response rate for completion of the questionnaire was $68.6 \%$ (273/396) and there was no difference in the response between the state and private sectors with rates of $67.7 \%(182 / 269)$ and $71.7 \%(91 / 127)$, respectively $(p=0.42)$.

Most of the participants were female, $\leq 50$ years old and were registered nurses. Of the registered nurses, $40.1 \%$ had formal ICU training. Of the total sample, 177 participants (70.5\%) did not have any formal ICU training; however, more than half of the respondents $(56.5 \%)$ had $>1$ year of experience working in an ICU. The sample appears to represent a broad sociocultural diversity, evidenced by the range of home languages spoken by participants. There were approximately equal proportions of isiZulu speakers across both sectors and a relatively higher proportion of Setswana and English speakers in the state and private sectors, respectively. Sample demographics are detailed in Table 1.

\section{Perceptions, opinions and personal beliefs about organ donation}

Two-thirds of participants expressed a willingness to donate their own organs after death $(172 / 268 ; 64.2 \%)$. English home-language nurses were more willing to donate compared with those of other language groups $(p=0.015)$. When asked to consider their personal choice regarding deceased organ donation, $51.1 \%$ of nurses felt that the most important person to make this decision should be themselves, while alive, rather than their next-of-kin. The response rate to this question was lower than for other questions (221/273; 19.0\% did not respond). This suggested that participants may not have considered organ donation previously or found it difficult to answer the question.

Respondents were predominantly of the Christian faith (172/249; 69.1\%), with African traditional religions being the second most commonly practised religion in the sample $(41 / 249 ; 16.5 \%)$. Christian participants were more highly qualified $(p=0.0026)$ and were more inclined to donate their organs after death $(p=0.028)$, compared with those of an African traditional religious faith.

Irrespective of their employment sector, age and qualification, approximately two-thirds of nurses $(168 / 266 ; 63.2 \%)$ felt that their personal beliefs did not influence advice given to patients and families regarding organ donation. However, when asked about how the staff in their unit felt about organ donation, only $36.8 \%$ (98/266) of participants felt positive about referring patients as potential organ donors. Older nurses $(>50$ years) were more positive than their younger colleagues $(p=0.1017)$ (Fig. 1).

\section{Referral for organ donation and scope of practice}

The majority of the participants $223 / 264(84.4 \%)$ felt it was the responsibility of the attending doctor to refer potential organ donors to the transplant procurement coordinator. However, older nurses ( $>50$ years), those with formal ICU training and English home-language speakers favoured nurse-based referrals when compared with their younger, less-qualified colleagues.

When asked whether they would follow a clear protocol - approved by their hospital and the Department of Health $(\mathrm{DoH})$ - that supports organ donor referral, most nurses $(216 / 269 ; 80.3 \%)$ said they would 


\begin{tabular}{|c|c|c|c|c|}
\hline & \multirow[b]{2}{*}{$\begin{array}{l}\text { Overall, } \\
n(\%)\end{array}$} & \multicolumn{2}{|c|}{ Sector } & \multirow[b]{2}{*}{$\begin{array}{l}p \text {-value for inter- } \\
\text { group test }\end{array}$} \\
\hline & & $\begin{array}{l}\text { State, } \\
n(\%)\end{array}$ & $\begin{array}{l}\text { Private, } \\
n(\%) \\
\end{array}$ & \\
\hline & $273(100)$ & $182(66.7)$ & $91(33.3)$ & \\
\hline Unit $^{*}$ & & & & $<0.0001$ \\
\hline ICU & $121(44.3)$ & 75 (46.9) & $46(50.4)$ & \\
\hline Casualty & $51(18.7)$ & $47(29.4)$ & $4(7.1)$ & \\
\hline High care & $24(8.8)$ & $18(11.3)$ & $6(10.7)$ & \\
\hline Ward & $20(7.3)$ & $20(12.5)$ & $0(0.0)$ & \\
\hline Other & $33(12.1)$ & $\mathrm{ND}$ & ND & \\
\hline \multicolumn{5}{|l|}{ Qualification $^{\dagger}$} \\
\hline $\mathrm{RN}$ & $112(41.0)$ & $73(42.4)$ & $39(49.4)$ & 0.73 \\
\hline ICU-trained RN & $75(27.5)$ & $53(30.8)$ & $22(27.8)$ & \\
\hline EN & $42(15.4)$ & $31(18.0)$ & $11(13.9)$ & \\
\hline NA & $16(5.9)$ & $15(8.7)$ & $7(8.9)$ & \\
\hline SN & $6(2.2)$ & ND & ND & \\
\hline CW & $1(0.4)$ & ND & ND & \\
\hline \multicolumn{5}{|c|}{ ICU experience of non-ICU-trained $\mathrm{RN}^{\ddagger}(n=177)$} \\
\hline$<3$ months & $40(22.6)$ & $26(25.2)$ & $14(27.5)$ & \\
\hline $3-6$ months & $8(4.5)$ & $21(20.4)$ & $6(11.8)$ & 0.56 \\
\hline $6-12$ months & $19(10.7)$ & & & \\
\hline $1-5$ years & $51(28.8)$ & $34(33.0)$ & $17(33.3)$ & \\
\hline$>5$ years & $36(20.3)$ & $22(21.4)$ & $14(27.5)$ & \\
\hline \multicolumn{5}{|l|}{ Age (years) } \\
\hline $20-30$ & 389 (13.9) & $28(15.9)$ & $10(11.6)$ & 0.37 \\
\hline $31-40$ & $113(41.4)$ & $70(39.8)$ & $43(50.0)$ & \\
\hline $41-50$ & $68(24.9)$ & $46(26.1)$ & $22(25.6)$ & \\
\hline $51-60$ & $38(13.9)$ & $32(18.2)$ & $11(12.8 \%)$ & \\
\hline $61-70$ & $5(1.8)$ & & & \\
\hline \multicolumn{5}{|l|}{ Genders } \\
\hline Female & $229(83.9)$ & $150(85.2)$ & 79 (92.9) & 0.075 \\
\hline Male & $32(11.7)$ & $26(14.8)$ & $6(7.1)$ & \\
\hline \multicolumn{5}{|l|}{ Home language\|l } \\
\hline isiZulu & $61(22.3)$ & $43(48.3)$ & $18(42.9)$ & $<0.0001$ \\
\hline English & $35(12.8)$ & $13(14.6)$ & $22(52.4)$ & \\
\hline Setswana & $35(12.8)$ & $33(37.1)$ & $2(4.8)$ & \\
\hline Sepedi & $29(10.6)$ & & & \\
\hline Sesotho & $22(8.1)$ & & & \\
\hline isiXhosa & $20(7.3)$ & & & \\
\hline Tsonga & $16(5.9)$ & & & \\
\hline Afrikaans & $14(5.1)$ & & & \\
\hline Venda & $10(3.7)$ & & & \\
\hline Siswati & $7(2.6)$ & & & \\
\hline Ndebele & $4(1.5)$ & & & \\
\hline $\begin{array}{l}\mathrm{RN}=\text { registered nurse; } \mathrm{IC} \\
{ }^{*} 24 \text { (8.8) not answered. } \\
{ }^{2} 21 \text { (7.7) not answered. } \\
{ }^{2} 23 \text { (13.0) not answered. } \\
{ }^{1} 11(4.0) \text { not answered. } \\
{ }_{1} 12(4.3) \text { not answered. } \\
{ }_{22}(7.3) \text { not answered. }\end{array}$ & istant; $\mathrm{SN}=\mathrm{st}$ & urse; $\mathrm{CW}=\mathrm{ca}$ & er; ND = not d & \\
\hline
\end{tabular}

abide by a protocol. This agreement was uniform across both sectors, regardless of qualification, age or home language.

In contrast, only $35.8 \%$ (97/271) of nurses felt that referring potential organ donors to a transplant coordinator was within their scope of practice and the remainder disagreed or were unsure, in equal proportions. Nurses who were older ( $>50$ years) $(p=0.0005)$ and English home-language speakers felt more confident that they could refer potential donors compared with other groups $(p<0.0001)$. Further, more qualified nurses felt significantly more confident that they could refer potential donors $(p<0.0001)$ (Fig. 2). This ranged from $50.7 \%$ for ICUtrained registered nurses to only $13.6 \%$ for student nurses.

Access to transplant services and perceptions of the role of transplant procurement coordinators

Most nurses (61.0\%) were aware that there was access to a procurement coordinator through their hospital, but more nurses in the private sector $(70.3 \%)$ were aware of this compared with those in the state sector (56.3\%; $p=0.0058)$. Across both sectors, younger, less-qualified 
nurses were least aware of this aspect. Of the respondents, $69.6 \%$ felt that they would be able to contact a procurement coordinator if required, but only the older and more qualified nurses knew whom to contact. Most participants indicated that the main roles of the procurement coordinator included obtaining consent from donor families, facilitating end- of-life discussions, reminding staff about the potential for organ donation, teaching the community about organ donation and tracing the family of a potential donor for consent (Fig. 3). Participants felt that the procurement coordinator should visit their units weekly. A noticeable difference was that more nurses in the state sector felt that coordinators should

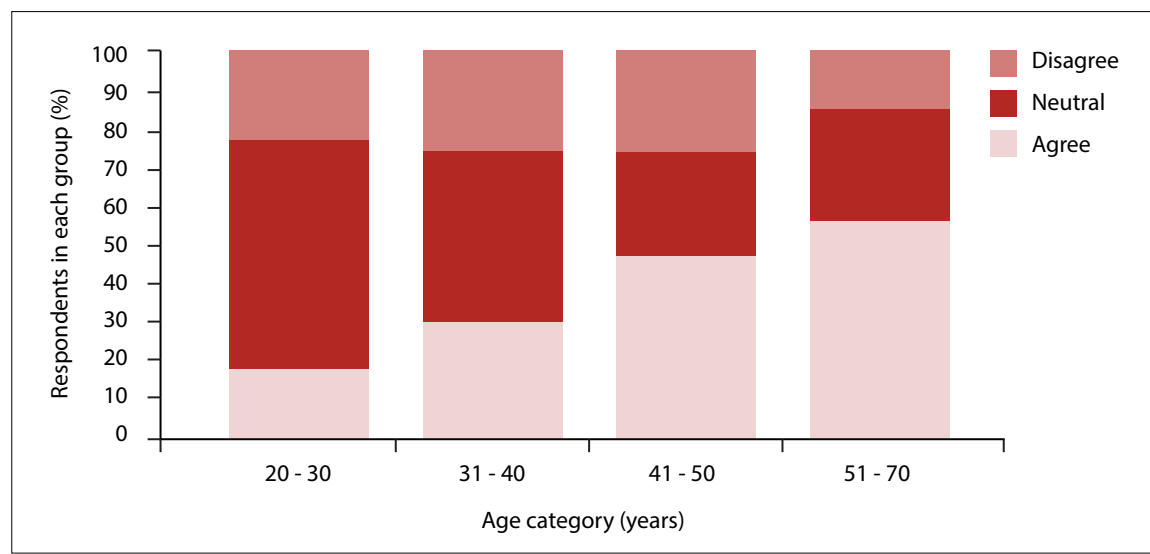

Fig. 1. Attitudes of staff members in the sampled units to organ donation.

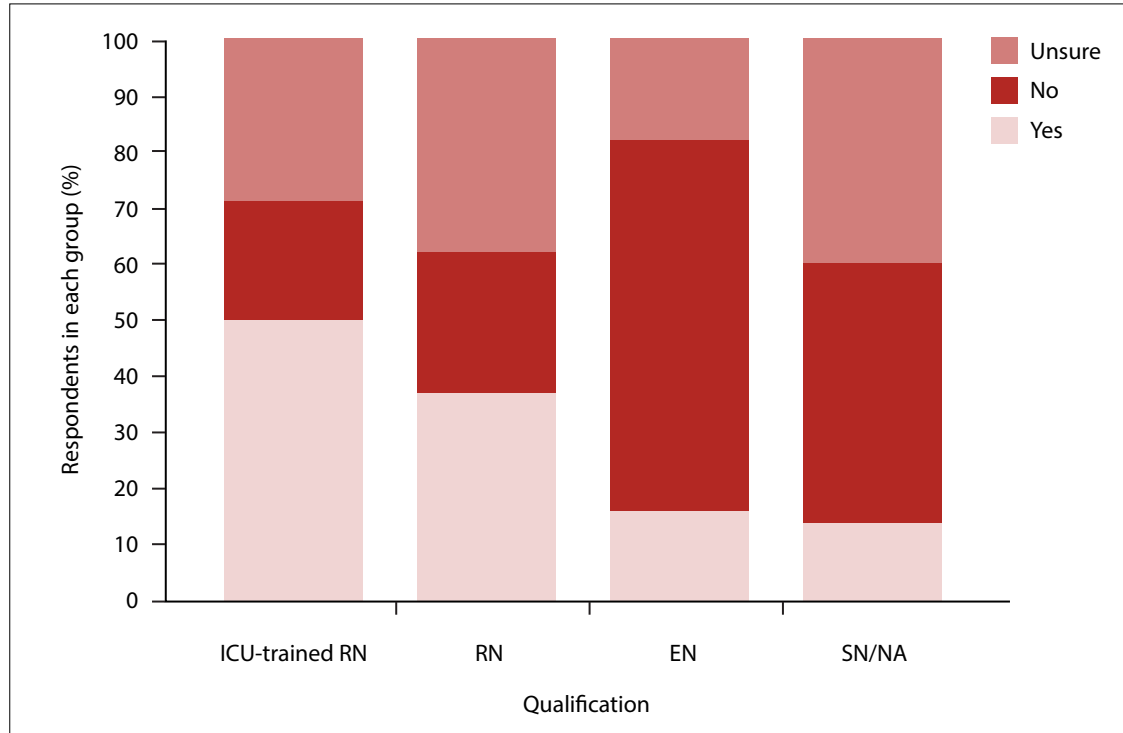

Fig. 2. Organ donation referrals and nursing scope of practice. (ICU = intensive care unit; $R N=$ registered nurse; $E N=$ enrolled nurse; $S N=$ student nurse; $N A=$ nurse assistant.)

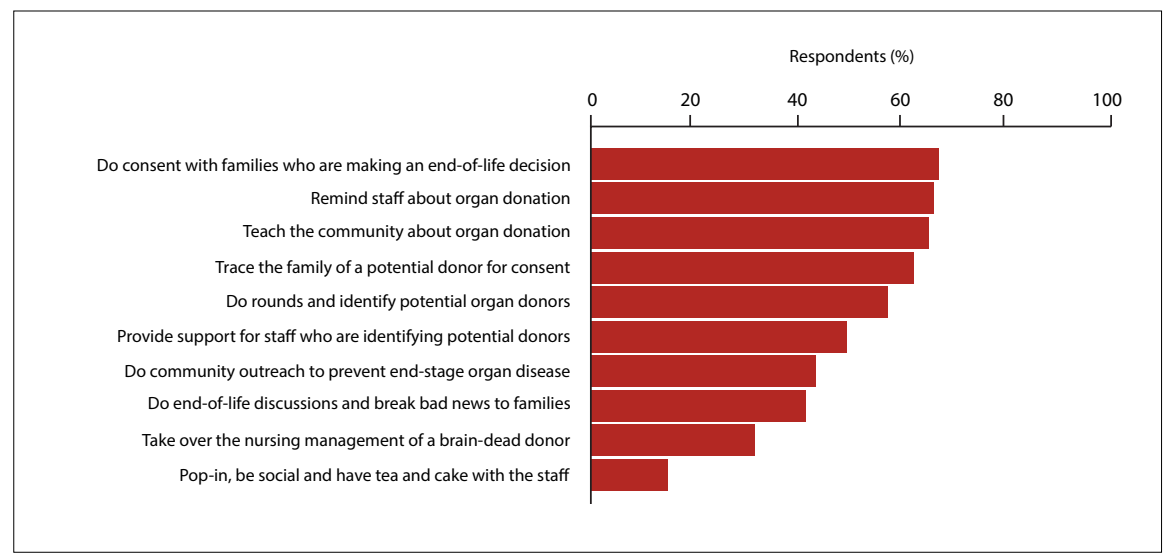

Fig. 3. Perceived role of the transplant coordinator. be involved with educating communities regarding prevention of end-stage kidney disease.

\section{Knowledge-based questions about organ procurement in South Africa}

The mean (standard deviation) knowledge score was 3.6 (1.3) (range 0 - 6). Knowledge regarding the supply of organs, access to transplants and legal rights was fair, whereas knowledge related to the allocation of organs and the use of organs from children and the elderly was poor (Fig. 4). The scores achieved by nurses in the state sector was lower compared with those in the private sector; ICU-trained nurses scored higher than enrolled nurses, and those who were willing to donate their organs after death scored higher than those who were unwilling to donate.

\section{Discussion}

This is the first quantitative study across the state and private sectors in SA showing that nurses expressed favorable attitudes to deceased organ donation; our findings indicated that the nurses' personal beliefs did not influence the donor referral process. Despite this, referral numbers in Johannesburg have been static for the past 5 years (K Crymble, Transplant Unit, Wits Donald Gordon Medical Centre, personal communication, July 2017). Our findings add to other SA studies which dispel perceptions that low referral rates are a function of personal opinions or beliefs that do not favour organ donation. ${ }^{[5,7]}$ Rather, our findings suggest that lack of a structured protocol is an endemic obstacle to deceased organ donor referral for nurses. Considering the finding that most nurses would willingly follow a structured referral protocol, this study presents good evidence for addressing low referral rates through the implementation of a protocol. Internationally, similar changes in legislation and guidelines have resulted in a significant increase in donor referrals. ${ }^{[7]}$

\section{Scope of practice and professional roles: Nurses and transplant procurement coordinators}

Our results reveal a lack of clarity regarding the roles of nurses and transplant procurement coordinators in organ donation in Johannesburg. Although nurses do have a formal scope of practice, it is vague and interpreting the role of the nurse in organ donation is challenging. 


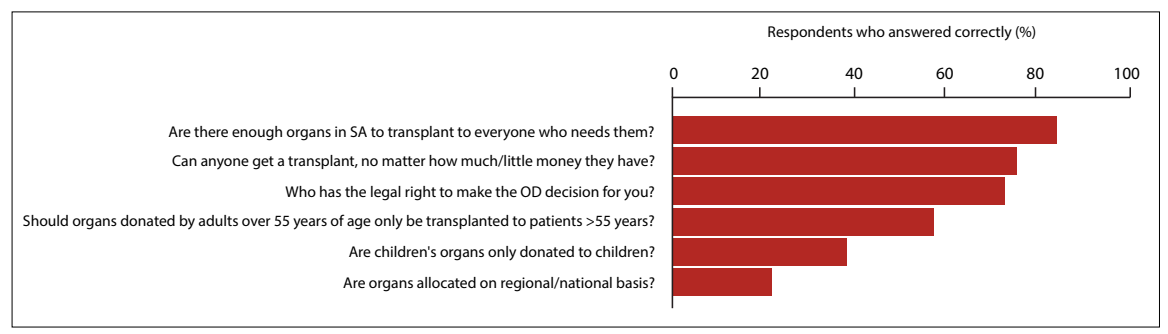

Fig. 4. Knowledge questions. $(\mathrm{OD}=$ organ donation. $)$

Table 2. Formal organ donation training for nurses and nursing students in Johannesburg*

\begin{tabular}{|c|c|}
\hline Qualification & Organ donation training \\
\hline Bachelor of Nursing (Undergraduate) & One 45 -minute session in the 4 th year \\
\hline Diploma in Nursing & Not covered \\
\hline $\begin{array}{l}\text { ICU specialist training through the } \\
\text { University of the Witwatersrand }\end{array}$ & One 45-minute session \\
\hline $\begin{array}{l}\text { Netcare and Mediclinic nursing } \\
\text { programmes }\end{array}$ & One 3 - 6-hour session on transplant \\
\hline $\begin{array}{l}\text { ICU }=\text { intensive care unit. } \\
{ }^{*} \mathrm{~A} \text { Hayward, personal communication, Novem }\end{array}$ & \\
\hline
\end{tabular}

Nursing scope of practice is legally defined within a set of parameters in SA; however, the specific roles required of nurses are not condensed into an exhaustive list because it is not feasible to accommodate every aspect of nursing management in a single document. This is particularly relevant with highly specialised and technology-intensive biomedicine which can be unpredictable in the role it requires of nurses. ${ }^{[8]}$

Previous SA and international research has found that the psychological and professional challenges facing nurses and doctors present major barriers to referral of potential organ donors. ${ }^{[5,10,11]}$ Owing to these challenges, formal donor referral guidelines that specify the role of nurses are warranted as a matter of urgency. The results of this study could be used to strongly advocate for, and inform, a protocol for mandatory donor referral that is nationally endorsed and supported by the DoH, the South African Nursing Council, the South African Transplant Society and all hospital groups.

Our results show that the role of the procurement coordinator is seen as varied and that nurses did not agree on exactly what their role was. While a procurement coordinator's duties would include securing consent from the organ donor's family, bedside donor care, family support, referral for transplantation and educating healthcare staff, there was a misperception that the role of the coordinator was to educate the public about disease prevention. This may be due to variations in the job description of transplant procurement coordinators across sectors and provinces, and in Johannesburg undertaking public education is not the primary responsibility of the procurement coordinator. Rather, it is the responsibility of donor education foundations, primarily the Organ Donor Foundation of SA. Confusion about the role of the coordinator is also evident in results suggesting that many of the research participants did not know how to contact the transplant procurement coordinator when there was a potential donor. This finding suggests that a more robust system for such communication is needed.

The roles and responsibilities for transplant procurement coordinators are defined in their employment contracts, and these vary widely across sectors and institutions, and may therefore differ depending on their employer and the region in which they are employed. Transplant coordinators play one of the most important roles in the organ donation process, so this ambiguity is a concern. It likely exacerbates doubts that nursing staff may experience about their role when liaising with transplant coordinators when there is a donor. International and SA research have shown that this type of uncertainty can hinder organ donation in a highly pressurised clinical environment where organisational routine and structured interaction are essential for its success. ${ }^{[5,12]}$

Our study revealed that the lack of knowledge about organ donation and organ donation may also be explained by a lack of formal organ donation education for nurses in the Johannesburg region. However, nurses in the private sector scored higher on the knowledge component of the questionnaire compared with those in the uncertainty about policies and roles in state sector. Because the private sector is more active when it comes to transplantation in Johannesburg, exposure to transplant on a regular basis, in practice, may also influence nurses' knowledge. In terms of national curricula, there is no formal obligation to teach organ donation and each educational institution chooses whether to include it at their discretion, with the approval of the nursing council. Table 2 summarises the paucity of formal organ donation education and training for nurses in Johannesburg.

The lack of formal organ donation education for nurses translates to a gap in patient management that should otherwise be standard practice. Furthermore, nurses have limited exposure to the practical aspects of organ donation, because transplantation is a lengthy and expensive procedure that takes place at a limited number of centres.

\section{Study limitations and strengths}

The limitations of this study were that it was conducted across Johannesburg and may not be generalisable to other regions in SA. Nurses who may not have felt sufficiently proficient in English could have self-excluded. A validated questionnaire was not used; however, there are no relevant, published, validated survey tools available for use in the SA setting.

The first advantage of this research is that the questionnaire, which has now been used in a large study, can be made available to other researchers. Secondly, the results of this quantitative study reflect the views of nurses across both private and state sectors in the correct ratio.

\section{Conclusion}

Our study found that there was a distinct lack of transplant protocol to guide nurses practising in Johannesburg, SA. Furthermore, there are misconceptions regarding the roles and responsibilities of nurses in the referral process. This creates an environment of uncertainty and insecurity for nurses who work in hospital units that may refer donors. Even when nurses reported positive attitudes, it appeared that the lack of protocol and defined guidelines prevented them from referring donors, probably because of the fear of retribution if a referral was perceived to be outside of their scope of practice. Future research should include a national extension of this study to other regions in SA.

The study presents evidence for the need for a nationally endorsed protocol that could guide nurses in donor referral. This 
is especially important given that nurses in Johannesburg said they would willingly adhere to such a protocol. It would be important for this protocol to emphasise teamwork and to clarify the role of doctors, as nurses need to be aware of how they might navigate relationships with doctors in the event that doctors themselves are unwilling to refer a potential donor. Regarding nursing education and training, it appears that substantial curriculum change is needed, and while it would have to be balanced with other priorities, some organ donation education at the undergraduate level could be helpful. At the level where nurses specialise by undertaking ICU training, organ donation education may be particularly beneficial, as these nurses could refer donors or care for recipients. While it is clear from the study that the coordinators do provide in-service training, this does not appear to be standardised and relatively few nurses access it because it is often confined to larger urban centres. A recognised nursing speciality training for transplant coordinators in SA, with a formal curriculum, should also be considered.

Acknowledgements. The authors would like to thank Heather Maher for database design and support and Victoria Masilo for her assistance with data capture. We are also very grateful to the Johannesburg nursing staff who participated in this study, with special thanks to the team of nurses in ward 565 at Charlotte Maxeke Johannesburg Academic Hospital who supported this project whole. We would like to pay tribute to every organ donor in SA, and to thank their families - without you there would not be life for others. We would also like to acknowledge all procurement coordinators countrywide for their commitment to ensuring compassionate care for all. We would like to thank Astellas Pharma (Pty) Ltd, SA and Wits Donald Gordon Medical Centre for financial support and we would particularly like to acknowledge to Astellas Pharma (Pty) Ltd, SA for their continued support of nurse-driven research in organ donation and transplant.

This paper is dedicated to the memory of Belinda Karen Rossi Britz (1966 - 2016).
Author contributions. KC: research design, ethics approval, data collection, data interpretation, feedback on drafts for submission. HRE: research design, ethics approval, data interpretation, paper drafting for submission (first and all subsequent drafts). JF: research design, ethics approval, data interpretation, substantial input on drafts for submission. PG: study design, piloted the study questionnaire, statistical analysis, feedback on drafts for submission.

Funding. This research was funded by an unrestricted educational grant from Astellas Pharma Pty (Ltd.) South Africa in conjunction with funding from Wits Donald Gordon Medical Centre.

Conflicts of interest. None.

1. Loveland J, Britz R, Joseph C, et al. Paediatric liver transplantation in Johannesburg revisited: 59 transplants and challenges met. S Afr Med J 2014;104(11):799-802.

2. Muller E, Barday Z, Mendwelson M, Kahn D. HIV-positive-to-HIV-positive kidney transplantation - results at 3 to 5 Years. N Engl J Med 2015;372(7):613-620. https://doi.org/10.1056/nejmoal408896

3. Donor Identification and Referral Strategy Group. Timely identification and referral of potential organ donors. Watford: NHS Blood and Transplant, 2014. www.odt.nhs.uk/pdf/timelyidentification-and-referral-potential-donors.pdf (accessed 11 July 2017).

4. Fabian J, Maher H, Bentley A, et al. Favourable outcomes for the first 10 years of kidney and pancreas transplantation at Wits Donald Gordon Medical Centre, Johannesburg, South Africa. S Afr Med J 2016;106(2):172-176. https://doi.org/10.7196/samj.2016.v106i2.10190

5. Etheredge HR, Penn C, Watermeyer J. Interprofessional communication in organ transplantation in Gauteng Province, South Africa. S Afr Med J 2017;107(7):615-620. https://doi.org/10.7196/ SAMJ.2017.v107i7.12355 https://doi.org/10.7196/SAMJ.2017.v107i7.12355

6. Harris PA, Taylor R, Thielke R, Payne J, Gonzalez N, Conde JG. Research electronic data capture (REDCap) - a metadata-driven methodology and workflow process for providing translational research informatics support. J Biomed Inform 2009;42(2):377-381. https://doi.org/10.1016/j. jbi.2008.08.010

7. Miranda B, Fernandez-Lucas M, De Felipe C, Naya M, Gonzalez-Posada J, Matesanz R. Organ donation in Spain. Nephrol Dial Transplant 1999;14:15-21.

8. N Geyer. Scope of nurses' practice. Prof Nurs Today 2016;20(1):51-52.

9. Wang Y-J, Lin C-Y. The experience of perioperative nurses involved in organ procurement. J Nurs Res 2009;17(4):278-285. https://doi.org/10.1097/jnr.0b013e3181c0038d

10. Greenhalgh T, Voisey C, Robb N. Interpreted consultations as 'business as usual'? An analysis of organisational routines in general practices. Sociol Health Ill 2007;29(6):931-954. https://doi org/10.1111/j.1467-9566.2007.01047.x

11. Becker MC. Organizational routines: A review of the literature. Ind Corp Change 2004;13(4):643678. https://doi.org/10.1093/icc/dth026

12. Naude A, Nel E, Uys H. Organ donation: Attitude and knowledge of nurses in South Africa. J Renal Care 2002;28(1):44-48. https://doi.org/10.1111/j.1755-6686.2002.tb00198.x

Accepted 28 August 2017. 